\title{
First hyperpolarizability of polymethineimine with long-range corrected functionals
}

\author{
Denis Jacquemin ${ }^{\mathrm{a}), \mathrm{b})}$ and Eric A. Perpète \\ Laboratoire de Chimie Théorique Appliquée, Group de Chimie-Physique Théorique et Structurale, \\ Facultés Universitaires Notre-Dame de la Paix, rue de Bruxelles, 61, B-5000 Namur, Belgium \\ Miroslav Medved' \\ Department of Chemistry, Faculty of Natural Sciences, Matej Bel University, Tajovského 40, \\ SK-97400 Banská Bystrica, Slovakia \\ Giovanni Scalmani and Michael J. Frisch \\ Gaussian, Inc., 340 Quinnipac Street, Building 40, Wallingford, Connecticut 06492 \\ Rika Kobayashi \\ ANU Supercomputer Facility, Leonard Huxley Building 56, Canberra ACT 0200, Australia \\ Carlo Adamo ${ }^{a), c)}$ \\ Ecole Nationale Supérieure de Chimie de Paris, Laboratoire Electrochimie et Chimie Analytique, \\ UMR CNRS-ENSCP no. 7575, 11, rue Pierre et Marie Curie, F-75321 Paris, Cedex 05, France
}

(Received 9 April 2007; accepted 24 April 2007; published online 21 May 2007)

\begin{abstract}
Using the long-range corrected (LC) density functional theory (DFT) scheme introduced by Iikura et al. [J. Chem. Phys. 115, 3540 (2001)] and the Coulomb-attenuating model (CAM-B3LYP) of Yanai et al. [Chem. Phys. Lett. 393, 51 (2004)], we have calculated the longitudinal dipole moments and static electronic first hyperpolarizabilities of increasingly long polymehtineimine oligomers. For comparison purposes Hartree-Fock (HF), Møller-Plesset perturbation theory (MP2), and conventional pure and hybrid functionals have been considered as well. HF, generalized gradient approximation (GGA), and conventional hybrids provide too large dipole moments for long oligomers, while LC-DFT allows to reduce the discrepancy with respect to MP2 by a factor of 3 . For the first hyperpolarizability, the incorrect evolution with the chain length predicted by HF is strongly worsened by BLYP, Perdew-Burke-Ernzerhof (PBE), and also by B3LYP and PBE0. On the reverse, LC-BLYP and LC-PBE hyperpolarizabilities are correctly predicted to be positive (but for the two smallest chains). Indeed, for medium and long oligomers LC hyperpolarizabilities are slightly smaller than MP2 hyperpolarizabilities, as it should be. CAM-B3LYP also strongly improves the B3LYP results, though a bit less impressively for small chain lengths. The present study demonstrates the efficiency of long-range DFT, even in very pathological cases. (C) 2007 American Institute of Physics. [DOI: 10.1063/1.2741246]
\end{abstract}

Polymethineimine (PMI) $\left[-(\mathrm{CH}=\mathrm{N})_{n}-\right]$ is the simplest asymmetric isoelectronic polymeric parent of polyacetylene (PA). Long PMI chains are characterized by very large first hyperpolarizabilities $(\beta),{ }^{1-3}$ and therefore they present a high potential for nonlinear optics (NLO) applications. However, an accurate evaluation of their NLO properties requires the inclusion of electron correlation (EC) effects that are extremely large, so large that they can even modify the sign of $\beta^{2,3} \mathrm{EC}$ can be introduced through wave function based approaches [i.e., Møller-Plesset perturbation theory (MP), coupled-cluster (CC), etc.] but such strategies usually require very large computational resources for long oligomers as the use of extended basis sets (BSs) is often essential. ${ }^{4}$ A more appealing alternative is apparently offered by the density functional theory (DFT). However, since the works of Champagne et al., it is well known that DFT calculations, using

\footnotetext{
${ }^{\text {a) }}$ Authors to whom correspondence should be addressed.

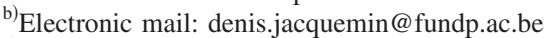

c) Electronic mail: carlo-adamo@enscp.fr
}

conventional functionals, fail to correctly predict the amplitude of NLO properties of long organic materials. ${ }^{5,6}$ The problem is related to the exchange part of the functional that suffers from short sightedness: the DFT screening of the polarization charge induced by the external electric field at the chain ends is far from being adequate. To circumvent the problem, several approaches have been applied to the calculation of (N)LO properties: current-DFT formalism, ${ }^{7}$ optimized effective potential for exact exchange, ${ }^{8,9}$ and the computationally less demanding long-range (LR) functionals (LR-DFT) ${ }^{10-15}$ [In the following, we use LR-DFT as a generic name for both long-range correlated (LC) and Coulomb-attenuating model (CAM) approaches.] Recently, the Hirao group has demonstrated that their LC approach can be a powerful tool to evaluate the $\beta$ of push-pull chains, ${ }^{13}$ as well as the second hyperpolarizability of PA. ${ }^{15}$ However, the evolution with the chain length of the $\beta$ of PMI is even more challenging because both Hartree-Fock (HF) and conventional hybrids provide the incorrect sign for the medium chain length. ${ }^{3}$ Indeed, the correct values are not bracketed by 
TABLE I. Longitudinal dipole moments of all-trans-polymethineimine chains. All values are in au and have been computed with the 6-31G $(d)$ basis set on the HF/6-31G $(d)$ geometry (see Ref. 3). The HF, MP2, B3LYP, and PBE0 results come from Ref. 3. At the bottom of the table, the extrapolated values per unit cell of the polymer $(\infty \pm \Delta \infty)$ are given.

\begin{tabular}{|c|c|c|c|c|c|c|c|c|c|}
\hline$n$ & $\mathrm{HF}$ & MP2 & BLYP & PBE & B3LYP & PBE0 & LC-BLYP & LC-PBE & CAM-B3LYP \\
\hline 2 & 0.525 & 0.360 & 0.333 & 0.331 & 0.372 & 0.382 & 0.367 & 0.371 & 0.389 \\
\hline 4 & 1.478 & 0.999 & 0.990 & 0.989 & 1.095 & 1.121 & 1.086 & 1.095 & 1.137 \\
\hline 6 & 2.713 & 1.820 & 1.866 & 1.867 & 2.058 & 2.105 & 2.035 & 2.049 & 2.130 \\
\hline 8 & 4.133 & 2.768 & 2.921 & 2.922 & 3.207 & 3.276 & 3.143 & 3.163 & 3.297 \\
\hline 10 & 5.673 & 3.806 & 4.123 & 4.123 & 4.496 & 4.586 & 4.358 & 4.386 & 4.584 \\
\hline 12 & 7.296 & 4.909 & 5.445 & 5.455 & 5.891 & 5.998 & 5.649 & 5.683 & 5.958 \\
\hline 14 & 8.975 & 6.059 & 6.862 & 6.876 & 7.364 & 7.485 & 6.994 & 7.035 & 7.394 \\
\hline 16 & 10.695 & 7.244 & 8.355 & 8.372 & 8.895 & 9.028 & 8.378 & 8.426 & 8.874 \\
\hline 18 & 12.445 & 8.455 & 9.905 & 9.926 & 10.470 & 10.611 & 9.790 & 9.844 & 10.387 \\
\hline 20 & 14.216 & 9.686 & 11.500 & 11.525 & 12.076 & 12.226 & 11.223 & 11.284 & 11.924 \\
\hline 22 & 16.003 & 10.930 & 13.128 & 13.158 & 13.707 & 13.863 & 12.672 & 12.740 & 13.479 \\
\hline 24 & 17.802 & 12.186 & 14.782 & 14.817 & 15.356 & 15.517 & 14.133 & 14.207 & 15.048 \\
\hline 26 & 19.611 & 13.449 & 16.457 & 16.496 & 17.020 & 17.185 & 15.602 & 15.683 & 16.628 \\
\hline 28 & 21.426 & 14.720 & 18.147 & 18.190 & 18.694 & 18.863 & 17.079 & 17.166 & 18.215 \\
\hline 30 & 23.247 & 15.996 & 19.849 & 19.897 & 20.377 & 20.549 & 18.561 & 18.655 & 19.808 \\
\hline$\infty$ & 0.925 & 0.654 & 0.893 & 0.899 & 0.867 & 0.868 & 0.756 & 0.761 & 0.816 \\
\hline$\Delta \infty$ & 0.015 & 0.013 & 0.034 & 0.025 & 0.021 & 0.023 & 0.009 & 0.011 & 0.013 \\
\hline
\end{tabular}

HF and B3LYP (or alike) results as it is the case for PA and push-pull systems: any simple HF/DFT combination seems to be doomed to failure. In this Communication, we test the efficiency of LC (Ref. 10) and CAM (Ref. 16) approaches for computing the electronic $\beta$ of PMI oligomers.

The methodological tools used in this Communication have been described in detail previously, ${ }^{2,3,14}$ and we consequently provide a short summary only. We performed all LC (Ref. 10) and CAM (Ref. 16) calculations with the latest developmental version of the GAUSSIAN package, ${ }^{17}$ while the remaining computations have been performed with GAUSSIAN03. ${ }^{18}$ We restrict ourselves to the $6-31 \mathrm{G}(d) \mathrm{BS}$ and the HF/6-31G $(d)$ geometries for all-trans-conformers, to allow calculations on long oligomers as well as straightforward comparisons with previously published data. ${ }^{3}$ We refer the interested reader to Refs. 3 and 19 for the discussion about EC and BS effects on these geometries. We have only considered longitudinal properties in this work, as this component (denoted by the $L$ subscript) clearly dominates the total response of PMI chains. ${ }^{20}$ The vibrational contribution ${ }^{21}$ to $\beta$ have been neglected at this stage; as a first description of the importance of this contribution in PMI is already available. $^{22}$ The MP2 dipole moment $\left(\mu_{L}\right)$ and $\beta_{L}$ have been determined through the numerical differentiation of the total energy computed under several external electric field strengths. ${ }^{2}$ The DFT $\mu_{L}$ have been obtained from the standard expectation value formula whereas DFT $\beta_{L}$ have been computed by using a finite-field procedure based on the numerical differentiation of the analytic polarizabilities evaluated with several electric field amplitudes. ${ }^{6}$ All DFT calculations have been performed with an ultrafine integration grid [pruned $(99,590)$ grid]. The properties reduced per unit cell are defined as $\Delta X_{L}(n)=\frac{1}{2}\left[X_{L}(n)-X_{L}(n-2)\right]$ with $X=\mu$ or $\beta$. The polymeric properties (per unit cell) have been obtained by extrapolating the oligomeric values. ${ }^{20}$

The dipole moments are listed in Table I and the evolu- tion with the chain length of $\Delta \mu_{L}(n)$ is sketched in Fig. 1. For the dipole moments, $\operatorname{CCSD}(\mathrm{T})$ calculations using very large BS reveal that MP2 values are only too big by about 5\%: The CCSD(T)/MP2 ratio are 0.96, 0.96, and 0.95 for $n=2,4$, and 6 , respectively. ${ }^{4}$ This conclusion is also supported by MP4 calculations using a smaller basis set. ${ }^{3}$ For chains longer than the tetramer, both $\mathrm{HF}$ and pure-GGA schemes overestimate $\mu_{L}$ by $+49 \%$ (HF) and $+8 \%$ (BLYP and PBE) for the decamer but by $+41 \%(\mathrm{HF})$ and $+37 \%$ (BLYP and PBE) for the polymer. Conventional hybrids

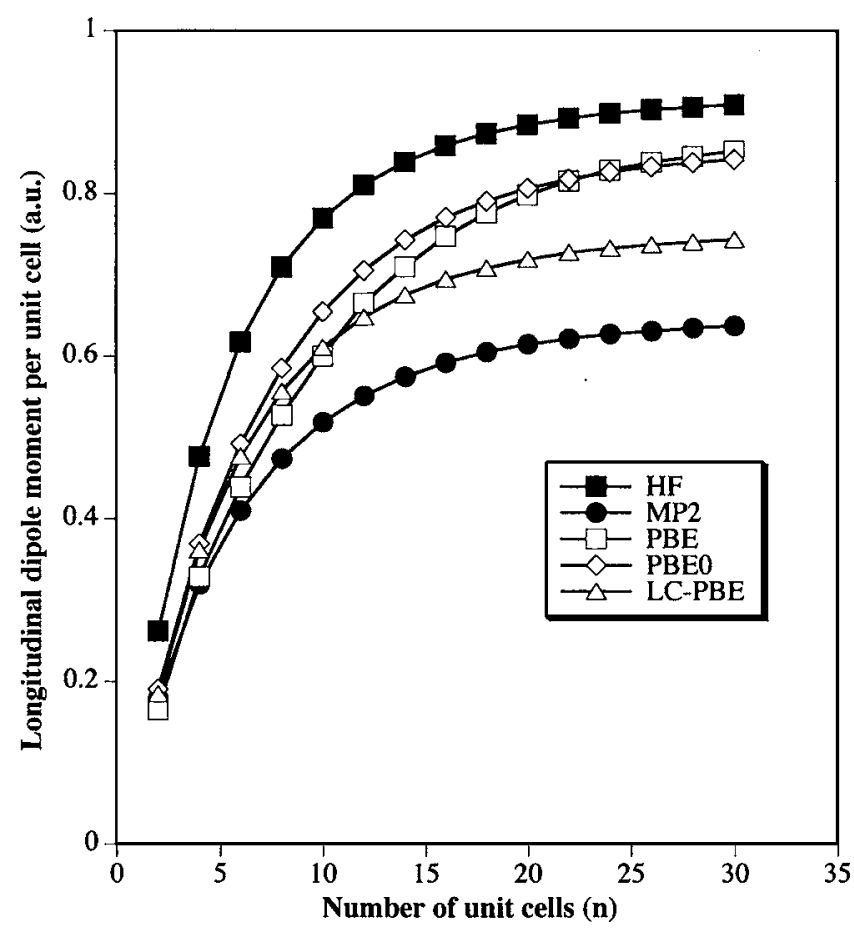

FIG. 1. Static longitudinal dipole moment per unit cell $\Delta \mu_{L}(n)$ for increasingly long polymethineimine chains. 
TABLE II. Longitudinal first hyperpolarizabilities of all-trans-polymethineimine chains. All values are in au and have been computed with the 6-31G $(d)$ basis set on the HF/6-31G $(d)$ geometry (see Ref. 3). The HF, MP2, B3LYP, and PBE0 results come from Ref. 3. At the bottom of the table, the extrapolated values per unit cell of the polymer $(\infty \pm \Delta \infty)$ are given.

\begin{tabular}{|c|c|c|c|c|c|c|c|c|c|}
\hline$n$ & $\mathrm{HF}$ & MP2 & BLYP & PBE & B3LYP & PBE0 & LC-BLYP & LC-PBE & CAM-B3LYP \\
\hline 2 & -56 & 64 & -33 & -36 & -33 & -36 & -16 & -20 & -25 \\
\hline 4 & -378 & 653 & -910 & -910 & -608 & -554 & -63 & -67 & -255 \\
\hline 6 & -912 & 2390 & -5930 & -5910 & -3109 & -2638 & 106 & 112 & -780 \\
\hline 8 & -1297 & 5890 & -21076 & -21023 & -8595 & -6829 & 1103 & 1138 & -1068 \\
\hline 10 & -1281 & 11494 & -52919 & -52866 & -16582 & -12824 & 3429 & 3513 & -343 \\
\hline 12 & -797 & 19192 & -104948 & -105011 & -25201 & -17458 & 7269 & 7422 & 1916 \\
\hline 14 & 122 & 28764 & -174642 & -175020 & -32306 & -20678 & 12575 & 12813 & 5875 \\
\hline 16 & 1408 & 39917 & -253619 & -254515 & -36397 & -21091 & 19185 & 19522 & 11459 \\
\hline 18 & 2991 & 52355 & -330870 & -332328 & -36820 & -18444 & 26903 & 27349 & 18486 \\
\hline 20 & 4810 & 65829 & -397059 & -399167 & -33611 & -12900 & 35539 & 36102 & 26739 \\
\hline 22 & 6813 & 80119 & -446940 & -449430 & -27088 & -4804 & 44920 & 45606 & 36010 \\
\hline 24 & 8960 & 95049 & & & -17710 & 5496 & 54899 & 55713 & 46111 \\
\hline 26 & 11218 & 110487 & & & -5941 & 17587 & 65359 & 66306 & 56887 \\
\hline 28 & 13562 & 126318 & & & & & 76199 & 77281 & 68203 \\
\hline 30 & 15975 & 142468 & & & & & 87349 & 88570 & 79960 \\
\hline$\infty$ & 1426 & 9171 & & & & & 7071 & 7148 & 7792 \\
\hline$\Delta \infty$ & 159 & 796 & & & & & 896 & 904 & 1004 \\
\hline
\end{tabular}

B3LYP and PBE0 do not improve the pure-GGA estimates $(+33 \%$ in the polymer). On the contrary, LC and CAM approaches are much more satisfying, although they still overshoot the longitudinal dipole by $+16 \%$ and $+25 \%$, respectively, when $n$ reaches $\infty$. For extended molecules the LC approach basically reduces the GGA error on $\mu_{L}$ by a factor of 2.3. In addition, the $\mathrm{LC} \mu_{L}$ are much more consistent with the MP2 results. Indeed, the LC-PBE curve almost parallels the MP2 line in Fig. 1: Between $n=6$ and $n=30$ the overestimation is always bracketed by the $+16 \%$ and $+18 \%$ limits [for $\left.\Delta \mu_{L}(n)\right]$ whereas in the same time the PBE error goes from $+7 \%$ to $+33 \%$.

PMI polarizabilities computed with LR-DFT have recently been published, ${ }^{14}$ and it has been found that both LC and CAM methodologies were very satisfying.

The $\beta_{L}$ computed within several computational schemes are compared in Table II and the corresponding evolution with the chain length of $\Delta \beta_{L}(n)$ is plotted in Fig. 2. The $\beta_{L}$ of the dimer, tetramer, and hexamer are positive at the MP and CC levels. ${ }^{4}$ In addition, $\operatorname{CCSD}(\mathrm{T})$ calculations demonstrate that the MP2 $\beta_{L}$ is too large by about $35 \%$ for the hexamer (this percentage apparently decreases with chain length). ${ }^{4}$ This is consistent with other MP4 estimates: ${ }^{2,3}$ All the MP2 $\beta_{L}$ systematically have the correct sign though their amplitude is too large by about $30 \%$ for medium chain lengths. The set of $\beta_{L}$ listed in Table II illustrates the doom of conventional DFT: For $n=12$, MP2 predicts a +19 $\times 10^{3}$ au $\beta_{L}$ whereas both GGA yield $-105 \times 10^{3}$ au, that is an error of $653 \%$. Full-range hybrids correct this behavior but to a much too small extent: $-25 \times 10^{3}$ au $(232 \%)$ with B3LYP and $-17 \times 10^{3}$ au (189\%) with PBE0. This glaringly appears on Fig. 2: BLYP and B3LYP actually accentuate the incorrect HF evolution instead of correcting it. Indeed, both functionals provide the wrong direction for the EC corrections to $\beta_{L}$. As up to $n=12$, both GGA and HF predict the incorrect sign for $\beta_{L}$; any straightforward combination is likely to be useless. On the contrary, LC and CAM accurately foresee the direction of the $\mathrm{EC}$ effects for all chain lengths. In addition, the LC-BLYP and LC-PBE $\beta_{L}$ and $\Delta \beta_{L}$ are only negative for the dimer and tetramer but positive for longer chains. These negative values for $n=2$ and $n=4$ are probably related to a (slightly) overestimated chain-end contribution, as in the HF model. ${ }^{2}$ The LC-BLYP and LC-PBE evolutions with chain length are also much more reasonable than that of B3LYP and PBE0: they parallel the MP2 curves but with smaller absolute values (Fig. 2). For the polymer the LC-BLYP $\Delta \beta_{L}$ is $23 \%$ smaller than the MP2 response, i.e., it

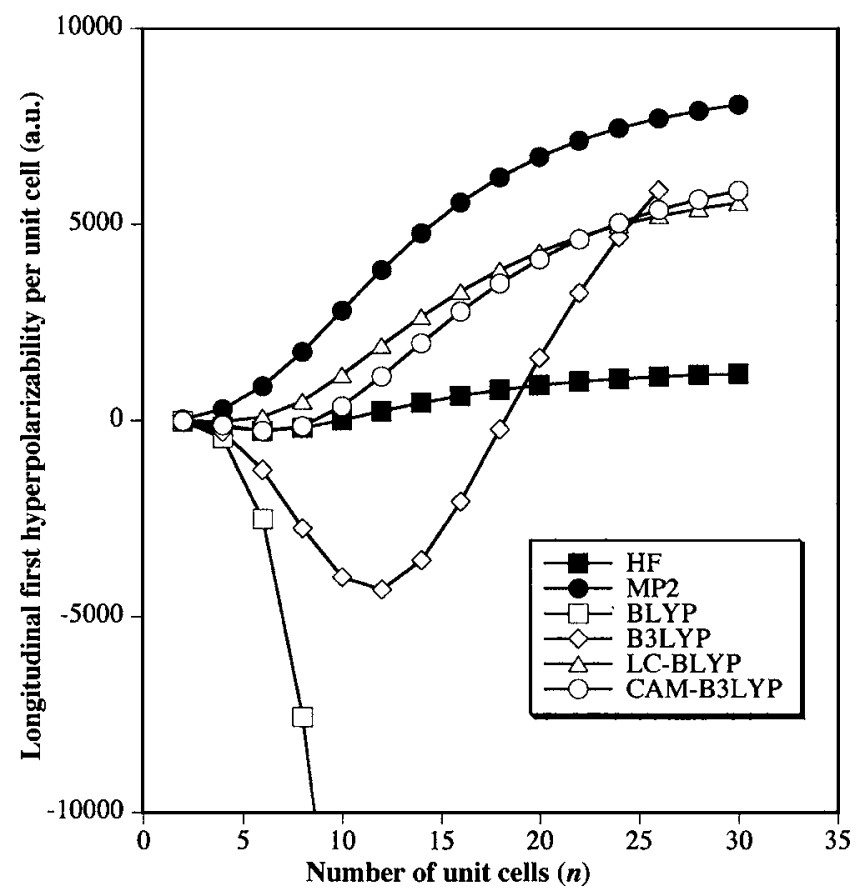

FIG. 2. Static longitudinal first hyperpolarizability per unit cell $\Delta \beta_{L}(n)$ for increasingly long polymethineimine chains. 
lies in the correct region, close to the values obtained by sophisticated post-HF (MP4 and CC) approaches. CAMB3LYP $\Delta \beta_{L}(n)$ are similar to HF for short chains, but qualitatively follows the MP2 curve when $n \geqslant 10$. Therefore, LRDFT schemes are much more efficient than GGA or conventional hybrids for evaluating the NLO properties of medium and large PMI chains. In addition, the convergence of the finite-field calculations is much easier with LR-DFT, allowing computations on more extended systems.

The longitudinal dipole moments and static electronic first hyperpolarizabilities of short, medium, and long polymethineimine chains computed with various DFT functionals have been compared to $\mathrm{HF}$ and MP2 results. It turns out that LC-DFT approximations significantly reduce the GGA error for the dipole moments whereas full-range hybrids only allow a small correction. Except for the dimer and tetramer, LC first hyperpolarizabilities are positive and slightly smaller than the MP2 values, indicating an accurate evolution with the chain length. This is a remarkable result; as up to $n=12$, $\mathrm{HF}, \mathrm{GGA}$, and conventional hybrids all provide the incorrect sign for $\beta_{L}$ : LC-DFT restores the correct balance between chain ends and unit cells contributions, ${ }^{3}$ although the correct values are not bracketed by the HF and GGA results. CAMB3LYP also significantly improves the B3LYP estimates, especially for $\beta_{L}$, though not as striking than LC for short chain lengths. Together with our previous investigation, ${ }^{14}$ this Letter shows that, for the few pathological cases in which traditional hybrid functionals become unsatisfying, LC and CAM functionals could probably allow a significant accuracy improvement.

Two of the authors (D.J. and E.A.P.) thank the Belgian National Fund for their research associate positions. Three of the authors (D.J. E.A.P., and C.A.) thank the Commissariat Général aux Relations Internationale and the Egide agency for supporting this work within the framework of the Tournesol Scientific cooperation between the Communauté Française de Belgique and France. One of the authors (M.M.) acknowledges the partial support from the Research and Development Agency of the Slovak Republic (Project No. APVT-20-009504). Non-LR-DFT calculations have been performed on the Interuniversity Scientific Computing Facil- ity (ISCF), installed at the Facultés Universitaires NotreDame de la Paix (Namur, Belgium), for which the authors gratefully acknowledge the financial support of the FNRSFRFC and the "Loterie Nationale" for the convention number 2.4578 .02 and of the FUNDP.

${ }^{1}$ D. Jacquemin, B. Champagne, and B. Kirtman, J. Chem. Phys. 107, 5076 (1997).

${ }^{2}$ D. Jacquemin, B. Champagne, and J. M. André, Chem. Phys. Lett. 284, 24 (1998).

${ }^{3}$ D. Jacquemin, J. M. André, and E. A. Perpète, J. Chem. Phys. 121, 4389 (2004).

${ }^{4}$ M. Medved, J. Noga, D. Jacquemin, and E. A. Perpète (unpublished).

${ }^{5}$ B. Champagne, E. A. Perpète, S. van Gisbergen, E. J. Baerends, J. G. Snijders, C. Soubra-Ghaoui, K. Robins, and B. Kirtman, J. Chem. Phys. 109, 10489 (1998).

${ }^{6}$ B. Champagne, E. A. Perpète, D. Jacquemin, S. van Gisbergen, E. Baerends, C. Soubra-Ghaoui, K. Robins, and B. Kirtman, J. Phys. Chem. A 104, 4755 (2000).

${ }^{7}$ M. van Faasen, P. L. Boeij, R. van Leeuwen, J. A. Berger, and J. G. Snijders, J. Chem. Phys. 118, 1044 (2003).

${ }^{8}$ F. A. Bulat, A. Toro-Labbé, B. Champagne, B. Kirtman, and W. Yang, J. Chem. Phys. 123, 014319 (2005).

${ }^{9}$ B. Champagne, F. A. Bulat, W. Yang, S. Bonness, and B. Kirtman, J. Chem. Phys. 125, 194114 (2006).

${ }^{10}$ H. Iikura, T. Tsuneda, T. Yanai, and K. Hirao, J. Chem. Phys. 115, 3540 (2001).

${ }^{11}$ E. Rudberg, P. Salek, T. Helgaker, and H. Agren, J. Chem. Phys. 123, 184108 (2005).

${ }^{12}$ H. Sekino, Y. Maeda, and M. Kamiya, Mol. Phys. 103, 2183 (2005).

${ }^{13}$ M. Kamiya, H. Sekino, T. Tsuneda, and K. Hirao, J. Chem. Phys. 122, 234111 (2005).

${ }^{14}$ D. Jacquemin, E. A. Perpète, G. Scalmani, M. J. Frisch, R. Kobayashi, and C. Adamo, J. Chem. Phys. 126, 144105 (2007).

${ }^{15}$ H. Sekino, Y. Maeda, M. Kamiya, and K. Hirao, J. Chem. Phys. 126, 014107 (2007).

${ }^{16}$ T. Yanai, D. P. Tew, and N. C. Handy, Chem. Phys. Lett. 393, 51 (2004).

${ }^{17}$ M. J. Frisch, G. W. Trucks, H. B. Schlegel et al., GAUSSIAN DVP, Revision F.01 (Gaussian, Inc., Wallingford, CT, 2006).

${ }^{18}$ M. J. Frisch, G. W. Trucks, H. B. Schlegel et al., Gaussian03, Revision C.02 (Gaussian, Inc., Wallingford, CT, 2004).

${ }^{19}$ D. Jacquemin, A. Femenias, H. Chermette, I. Ciofini, C. Adamo, J. M. André, and E. A. Perpète, J. Phys. Chem. A 110, 5952 (2006).

${ }^{20}$ B. Champagne, D. Jacquemin, J. M. André, and B. Kirtman, J. Phys. Chem. A 101, 3158 (1997).

${ }^{21}$ M. Torrent-Sucarrat, M. Solà, M. Duran, J. M. Luis, and B. Kirtman, J. Chem. Phys. 118, 711 (2003).

${ }^{22}$ E. A. Perpète, B. Champagne, and D. Jacquemin, J. Mol. Struct.: THEOCHEM 529, 65 (2000). 